\title{
Characterization of Malassezia spp. in Oral Cavity of Dog
}

\author{
Takao Yoshikawa
}

Department of Microbiology and Immunology, Nihon University School of Dentistry at Matsudo, Matsudo, Chiba 271 -8587, Japan

Correspondence to :

Takao Yoshikawa

E-mail : yoshikawa1975@k3.dion.ne. jp

Keywords :

animal, fungus, Malassezia, dog

\begin{abstract}
The purpose of this study was to identify and to characterize of Malassezia spp. in the oral cavity of dog. One hundred and seventeen strains of Malassezia spp. were isolated from fresh clinical isolates of fungi from 20 dogs. The isolates grew well on Sabouraud agar, and produced catalase and precipitates on Tween-medium, and hydrolyzed esculin. Colony morphologies and sizes were characteristic on CHROMagar Malassezia (CHROM): they developed small, violet color, medium, violet color with pink edge, or large, pale pink color colonies. Molecular investigations of 18 isolates in three different morphological colonies on CHROM from 6 subjects were carried out by chitin synthase 2 (CHS2) gene sequence analysis. The results showed that isolates constitute three genetic types $\left(A_{c}, B_{c}\right.$, and $\left.C_{c}\right)$. Type $A_{c}, B_{c}$, and $C_{c}$ consisted of 3,8 , and 7 isolates, respectively. The three isolates from only one subject were grouped into the same genotype. These results suggest that the isolates in the oral cavity of dog were Malassezia pachydermatis, and the isolates in CHS2 genetic group were diverse. The relationship between colonial morphology and genetic typing was inconsistent.
\end{abstract}

\section{Introduction}

Fungi are recognized as one of the most frequent causes of opportunistic infections in humans (1). Currently, the presence of Malassezia spp. in oral cavity of dogs was reported (2). Malassezia spp. are fungi that have been recognized as members of the microbiological flora of human and animal skin (3, 4). Skin bacteria of licked areas may come to inhabit oral cavity and become involved in these infections. Dogs are close companions in many human families, and the owners are in close contact with these dogs. As the potential for exposure of the owner to the organism is therefore marked, handwashing practices and maintaining a distance with the dog may effectively eliminate the transmission of malassezia yeast to humans.

Extensive research on dogs will help clarify that an understanding of the microbial ecology of the mouth is fundamental to elucidating the etiology of most oral diseases. The purpose of the present investigation was to identify and characterize Malassezia spp. from the oral cavity of dogs.

\section{Materials and Methods}

\section{Organisms and media}

One hundred and seventeen fresh clinical isolates of Malassezia obtained from the oral cavity of 20 dogs, as described previously (2), as well as type strains of Malassezia pachydermatis JCM 10131 were used in this study. Strains of Malassezia were maintained on Sabouraud agar (SDA, Nissui Co., Tokyo, Japan). The following special media were used in this study. SDA, SDA-Tween, CHROMagar Malassezia (CHROM, CHROMagar, France) (5), modified CHROMagar Malassezia (modified CHROM) (5), Cremophor EL (Sigma, St. Louis, MO) agar (EL slant) (5), and Tween 60-esculin agar (TE slant) (5).

\section{Phenotypic feature testing}

The "typical phenotypic features" of Malassezia spp. were defined as shown in Table 1. All isolates of 
Table 1. Colonial morphology on CHROM

\begin{tabular}{cccc}
\hline \multirow{2}{*}{$\begin{array}{c}\text { Number of } \\
\text { subjects }\end{array}$} & \multicolumn{3}{c}{ Size and color of colony } \\
\cline { 2 - 4 } & $\mathrm{S}^{*}$ & $\mathrm{M}$ & $\mathrm{L}$ \\
\hline 6 & + & + & + \\
4 & + & - & - \\
3 & + & + & - \\
2 & + & - & + \\
1 & - & + & - \\
2 & - & + & + \\
2 & - & - & + \\
\hline
\end{tabular}

+ , Detection; *, See in the text

Malassezia were inoculated onto CHROM and special media (modified CHROM, SDA, SDA-Tween, EL slant, and TE slant) and incubated at $32{ }^{\circ} \mathrm{C}$ for 5 days before observation. SDA was used to determine the isolates' lipid dependence, SDA-Tween and modified CHROM for the production of precipitin from Tween, EL slants for the isolates' abilities to utilize polyethoxylated castor oil (Sigma-Aldrichi, Co., St. Louis, MO, USA), and TE slants for the isolates' abilities to hydrolyze esculin and utilize Tween 60 . Fresh cultures grown on CHROM were subjected to catalase test with $3 \%$ hydrogen peroxide. The colony size on CHROM was determined by measuring wellisolated single colonies and assessed as small $(<1$ $\mathrm{mm}$ ), medium (1 to $3 \mathrm{~mm}$ ), or large (3 to $5 \mathrm{~mm}$ ).

\section{Molecular analysis}

DNA was extracted from fungal cultures using the Promega Genome kit (Promega, Madison, WI, USA) according to the manufacturer's instructions. The regions of the large subunit (LSU) and internal transcribed space 1 (ITS-1) of nuclear ribosomal DNA and the chitin synthase 2 (CHS2) gene were sequenced directly form PCR products using the primer pairs of F63 and LR3 (6), 18SF1 and 5.8SR1 (7), and CED1 and CED2 (8), respectively. The PCR product was sequenced on an ABI PRIMS 310 genetic analyzer according to the manufacturer's instructions (Applied Biosystems, Foster City, CA, USA). The sequences for individual genes were compared with respective nucleotide sequences from reference strains of Malassezia. The closest known relatives of the new isolates were identified by searching databases. The gene sequences were compared with those available at the DNA Data Bank of Japan (DDBJ) using BLAST algorithm software.

\section{Results}

Phenotypic features

All isolates and type strain of $M$. pachydermatis grew after incubation at $32{ }^{\circ} \mathrm{C}$ for 4 days on CHROM. The colony size on CHROM was measured from well -isolated single colonies, and isolates were divided into three groups: small, violet color (S), medium, violet color with pink edge (M), and large, pale pink color (L) (Fig. 1A).

Table 1 shows the detection number of 3 different colonies from each dog. Six of 20 dogs had 3 kinds of strain. The most common strain was the $\mathrm{S}$ form. Precipitate appeared after days 2 and 3 and over 5 days of incubation, precipitate become more pronounced, and granularity and a zone of clearing were seen directly adjacent to the microbial growth on SDA-Tween (Fig. 1B) or modified CHROM.

All isolates and type strain grew on the lipid-free culture medium (SDA), and the biological feature of M. pachydermatis was specific (Table 2). All isolates

A
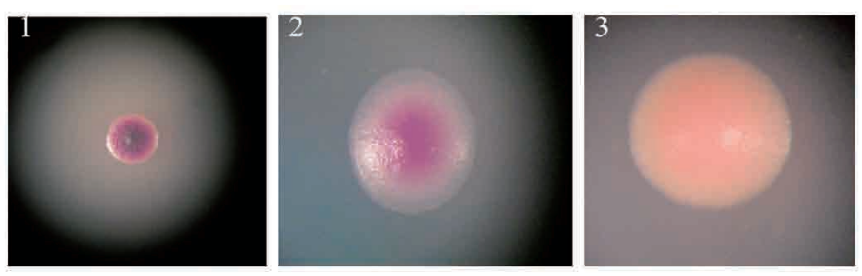

B

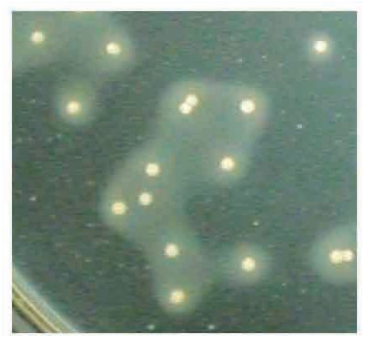

Fig. 1. Malassezia spp. colony on CHROM medium (A) and precipitate production on SDA-Tween medium (B) after 4 days incubation at $32{ }^{\circ} \mathrm{C}$. Numbers indicate each colony, as shown below: 1 , small and violet; 2 , medium and violet with pink edge ; 3 , large and pink. 
Table 2. Biological characterization based on the identification kit for Malassezia species

\begin{tabular}{lccc}
\hline & M. pachydermatis & M. furfur & $\begin{array}{c}\text { Other Malassezia } \\
\text { species }\end{array}$ \\
\hline Growth on & & & \\
Modified CHROM & $\mathrm{GP}$ & $\mathrm{G}$ & $\mathrm{G} / \mathrm{GP}$ \\
SDA & $\mathrm{G}$ & $\mathrm{N}$ & $\mathrm{N}$ \\
TE slant & $\mathrm{GB}$ & $\mathrm{GB}$ & $\mathrm{N} / \mathrm{GN} / \mathrm{GB}$ \\
EL slant & $\mathrm{G}$ & $\mathrm{G}$ & $\mathrm{N}$ \\
Catalase reaction & Positive & Positive & Negative \\
\hline
\end{tabular}

G, growth; N, no growth; GP, growth and production of precipitate ; $\mathrm{GB}$, growth and black zone; GN, growth and no change

grew on the EL slants medium. All isolates showed a positive catalase reaction, and black products in the medium were due to esculin hydrolysis products and ferrous iron in TE slants. These biological results concluded that the isolates were identified as $M$. pachydermatis.

\section{Molecular analyses}

Three kinds of typical isolate (NUM 155-S, -M, and $-\mathrm{L}$ ) from one subject and the JCM 10131 strain were analyzed by genetically. The amplification of DNA from clinical isolates with LSU, and ITS-1 primers yielded fragments of about 640, and $280 \mathrm{bp}$, respectively. These products were sequenced and subjected to a comparative analysis. The sequence relatedness was similar to that of $M$. pachydermatis with more than 99\% homology. The genotype of these products was further determined. The sequencing of amplicons representing the JCM 10131 strain and isolate NUM $155-S$ revealed $A_{L}$ and $A_{I}$, and $B_{L}$, and $B_{I}$ sequence types all for LSU and ITS-1, respectively (Table 3). The isolates NUM 155-M and NUM 155-L were grouped into type $\mathrm{C}_{\mathrm{L}}$ for LSU. In case of ITS-1 gene, NUM 155-M and NUM 155-L isolates were grouped into subtype $\mathrm{C}_{\mathrm{I}}$ and $\mathrm{C}_{2}$, respectively.

Eighteen isolates in three different morphological colonies on CHROM from 6 subjects and $M$. pachydermatis JCM 10131 were further analyzed by CHS2 gene sequencing. The results showed that isolates constitute three genetic types $\left(\mathrm{A}_{\mathrm{C}}, \mathrm{B}_{\mathrm{C}}\right.$, and $\mathrm{C}_{\mathrm{C}}$ ). Strain JCM 10131 was included in type $\mathrm{A}_{\mathrm{C}}$.
Table 3. Genetic sequence types of isolates and $M$. pachydermatis JCM 10131 for LSU, and ITS-1 genes

\begin{tabular}{lcc}
\hline Strain & LSU & ITS-1 \\
\hline M. pachydermatis JCM 10131 & $\mathrm{A}_{\mathrm{L}}$ & $\mathrm{A}_{\mathrm{I}}$ \\
Isolate & & \\
NUM 155-S & $\mathrm{B}_{\mathrm{L}}$ & $\mathrm{B}_{\mathrm{I}}$ \\
NUM 155-M & $\mathrm{C}_{\mathrm{L}}$ & $\mathrm{C} 1_{\mathrm{I}}$ \\
NUM 155-L & $\mathrm{C}_{\mathrm{L}}$ & $\mathrm{C} 2_{\mathrm{I}}$ \\
\hline
\end{tabular}

Table 4. CHS2 sequence types of isolates from dog oral cavity with reference strain M. pachydermatis JCM 10131

\begin{tabular}{|c|c|c|c|}
\hline & Type $A_{c}$ & Type $B_{c}$ & Type $\mathrm{C}_{\mathrm{c}}$ \\
\hline M. pachydermatis & JCM 10131 & & \\
\hline \multicolumn{4}{|c|}{ Isolates from subject } \\
\hline NUM 155 & & $155-\mathrm{S}$ & $155-\mathrm{M}, 155-\mathrm{L}$ \\
\hline NUM 2 & $2-\mathrm{S}$ & $2-\mathrm{M}$ & $2-\mathrm{L}$ \\
\hline NUM 4 & & & $4-\mathrm{S}, 4-\mathrm{M}, 4-\mathrm{L}$ \\
\hline NUM 5 & $5-\mathrm{M}$ & $5^{-} \mathrm{S}, 5^{-} \mathrm{L}$ & \\
\hline NUM 9 & $9-\mathrm{S}$ & 9-M, 9-L & \\
\hline NUM 16 & & $16-\mathrm{S}, 16-\mathrm{L}$ & $16-\mathrm{M}$ \\
\hline
\end{tabular}

Types $A_{c}, B_{c}$, and $C_{c}$ consisted of 3,8 , and 7 isolates (Table 4). The three isolates from subject 4 were grouped in the same genotype. However, isolates from subjects 155 and 16 were shared by two types, $\mathrm{B}_{\mathrm{C}}$ and $\mathrm{C}_{\mathrm{c}}$, and isolates from subjects 5 and 9 were shared by $A_{c}$ and $B_{c}$ types. NUM 2 isolates were grouped into three different types (Table 4).

\section{Discussion}

Members of the genus Malassezia are among the microbiological flora of the skin of homoeothermic animals, and known to be causative agents of pityriasis versicolor and seborrheic dermatitis (3, 4). Zoonotic transfer has been documented from dogs to immunocompromised patients by healthcare workers who own dogs (9). This species has been reported as a commensal on the skin of dog owners (9) and a causative organism of granulomatous skin infection in a dog owner (10). The genus Malassezia contains ten species classified as lipid-dependent and one, $M$. pachydermatis, which does not require lipid supplementation for growth $(11,12)$. The identification of members of the genus Malassezia is based on lipid 
dependency, as well as morphological, physiological, and/or molecular characteristics $(11,12)$. However, the identification of individual Malassezia species is more difficult because carbon assimilation and fermentation techniques are not applicable to this genus.

Selective media are very important to identify isolates from clinical samples. For human fungi isolation, CHROMagar Candida is widely used as an isolation medium on which colonies of the genus Candida can be distinguished by their color. CHROM is modified CHROMagar Candida for the genus Malassezia, and it was used as the primary culture medium. SDA was used to determine the lipid dependence of $M$. pachydermatis. EL slants were employed to determine the ability to utilize polyethoxylated castor oil of $M$. furfur and $M$. pachydermatis. TE slants were used to determine the ability to hydrolyze esculin. The isolates from the dog oral cavity exhibited all biological characteristics to grow on SDA, produce catalase and precipitin from SDATween or modified CHROM medium, and hydrolyze esucline. It is likely that the appearance of precipitate is due to the production of insoluble free fatty acids from lipid sources contained in the medium (13). These observations suggest differences in the lipase enzyme systems in Malassezia species (13). The production of precipitate on the lipid-medium may be of use in the identification of Malassezia isolates.

Chitin is one of the major structural componets of the fungal cell wall, and chitin synthases are membrane-bound proteins responsible for the catalytic polymerization of N-acetylglucosamine from UDPsugar (14). Fungal chitin synthases are encoded by CHS genes. CHS2 gene analysis confirmed three types $\left(\mathrm{A}_{\mathrm{C}}, \mathrm{B}_{\mathrm{C}}\right.$, and $\left.\mathrm{C}_{\mathrm{C}}\right)$ of $M$. pachydermatis isolates (15). Type $\mathrm{A}_{c}$ M. pachydermatis was predominant, and originates from lesions of dog and cat skin (16). Type $\mathrm{B}_{\mathrm{C}}$ was genetically close to the lipid-dependent Malassezia species, M. furfur (16). The isolates from the oral cavity were grouped into types $A_{c}, B_{c}$, and $\mathrm{C}_{\mathrm{c}}$ in this study (Table 4). Makimura et al (6). reported that the ITS-1 sequences of $M$. pachydermatis were close to those of M. furfur and M. obtuse. Such results suggest that the idea that the evolutionary differentiation of Malassezia spp. arose through adaptations for increasing dependency on exogenous lipids should be reconsidered.

This is the first report on M. pachydermatis identified in samples from the oral cavity of dogs. In this study the results suggest that the isolates from the oral cavity of dogs were identified as $M$. pachydermatis, and isolates in the CHS2 genetic group were diverse. The relationship between colonial morphology and genetic typing remains inconsistent.

\section{Acknowledgments}

The author is grateful to Professor M. Hirasawa for his direction and helpful advice, and appreciate Professors T. Ikemi and J. Mega for reviewing the manuscript. I thank Dr. K. Takada for her continuous and kind support. This study was supported in part by a Grant-in-Aid for SBFSR by MEXT 200812 .

\section{References}

1. Reichart PA, Samaranayake LP, Philipsen HP: Pathology and clinical correlates in oral candidiasis and its variants: a review. Oral Dis, $6: 85-91,2000$.

2. Kobayashi C: Distribution of yeast-form fungi in oral cavity of dog. Int J Oral-Med Sci, 7 : 40-44, 2008.

3. Ashbee HR, Evans EG: Immunology of diseases associated with Malassezia species. Clin Microbiol Rev, 15: 21-57, 2002.

4. Guého E, Boekhout T, Ashbee HR, Guillot J, Van Belkum A, Faergemann J: The role of Malassezia species in the ecology of human skin and as pathogens. Med Mycol, 36 : 220-229, 1998.

5. Kaneko T, Makimura K, Sugita T, Yamaguchi H : Tween 40-based precipitate production observed on modified chromogenic agar and development of biological identification kit for Malassezia species. Med Mycol, 44 : 227-231, 2006.

6. Fell JW, Boekhout T, Fonseca A, Scorzetti G, Statzell -Tallman A : Biodiversity and systematics of basidiomycetous yeasts as determined by largesubunit rDNA D1/D2 domain sequence analysis. Int J Syst Evol Microbiol, 50 : 1351-1371, 2000.

7. Makimura K, Tamura Y, Kudo M, Uchida K, Saito H, Yamaguchi H : Species identification and strain typing of Malassezia species stock strains and clinical isolates based on the DNA sequences of nuclear ribosomal internal transcribed spacer 1 regions. J Med 
Microbiol, 49 : 29-35, 2000.

8. Cafarchia C, Latrofa MS, Testini G, Parisi A, Guillot J, Gasser RB, Otranto D : Molecular characterization of Malassezia isolates from dogs using three distinct genetic markers in nuclear DNA. Mol Cell Probes, 21 : 229-238, 2007.

9. Morris DO, O'shea K, Shofer FS, Rankin S: Malassezia pachydermatis carriage in dog owners. Emerg Infect Dis, 11: 83-88, 2005.

10. Fan YM, Huang WM, Li SF, Wu GF, Lai K, Chen RY : Granulomatous skin infection caused by Malassezia pachydermatis in a dog owner. Arch Dermatol, 142 : 1181-1184, 2006.

11. Guého E, Midgley G, Guillot J : The genus Malassezia with description of four new species. Antonie van Leeuwenhoek, 69: 337-355, 1996.

12. Guillot J, Guého E, Lesourd M, Midgley G, Chevrier G,
Dupont B: Identification of Malassezia species. A practical approach. J Mycol Med, 6 : 103-110, 1996.

13. Hammer KA, Riley TV : Precipitate production by some Malassezia species on Dixon's agar. Med Mycol, 38: 105-107, 2000.

14. Bulawa CE: Genetics and molecular biology of chitin synthesis in fungi. Ann Rev Microbiol, 47: 505-534, 1993.

15. Aizawa T, Kano R, Nakamura Y, Watanabe S, Hasegawa A : Molecular heterogeneity in clinical isolates of Malassezia pachydermatis from dogs. Vet Microbiol, 70: 67-75, 1999.

16. Aizawa T, Kano R, Nakamura Y, Watanabe S. Hasegawa A: The genetic diversity of clinical isolates of Malassezia pachydermatis from dogs and cats. Med Mycol, 39 : 329-334, 2001. 